\title{
The Mass of the White Dwarf in the Recurrent Nova CI Aquilae
}

\author{
D. I. Sahman and V. S. Dhillon \\ Department of Physics and Astronomy, University of Sheffield, S3 7RH, UK. \\ email: d.sahman@sheffield.ac.uk
}

\begin{abstract}
If recurrent novae (RNe) are progenitors of Type Ia Supernovae (SNe Ia), their white dwarfs (WD) must have masses close to the Chandrasekhar limit. The most reliable means of determining WD masses in RNe is dynamically, via radial-velocity and rotational-broadening measurements of the companion star. Such measurements require the system to be both eclipsing and to show absorption features from the secondary star. The only other reliable RNe mass estimate is for U Sco, which has a WD mass of $1.55 \pm 0.24 \mathrm{M}_{\odot}$ (Thoroughgood et al. 2001).

We present new time-resolved, intermediate-resolution spectroscopy of the eclipsing RN CI Aql during quiescence. We find the mass of the WD to be $1.02 \pm 0.08 \mathrm{M}_{\odot}$ and the mass of the secondary star to be $2.41 \pm 0.2 \mathrm{M}_{\odot}$. We estimate the radius of the secondary to be $2.10 \pm 0.07 \mathrm{R}_{\odot}$. The high mass ratio of $\mathrm{q}=2.37 \pm 0.15$ and the high secondary star mass suggests that mass transfer occurs on a thermal timescale. We suggest that CI Aql is evolving into a supersoft x-ray source, and ultimately will explode as an SN Ia.
\end{abstract}

Keywords. stars: novae, cataclysmic variables, individual (CI Aquilae)

\section{Introduction}

$\mathrm{RNe}$ are cataclysmic variables $(\mathrm{CVs})$ that have had more than one recorded nova outburst. Despite the mass loss from the nova outburst, the WD is believed to have a net increase in mass. When the WD reaches the Chandrasekhar limit, it begins carbon burning in its degenerate core. This leads to a thermonuclear runaway which totally disrupts the WD and results in an SN Ia explosion (Hachisu \& Kato 2001).

The second recorded outburst of CI Aql in May 2000 secured its place amongst the few known RNe, after its first outburst in 1917. Schaefer (2001) also found archival evidence of a further outburst in 1941. CI Aql shows eclipses on an orbital period of 14.8 hours, and has a quiescent magnitude of $V \sim 15.7$. We seek to use time-resolved spectroscopy of CI Aql to determine the radial velocity, rotational velocity and inclination of the system, which can then be used to accurately derive the masses of the two component stars.

\section{Observations}

We obtained 186 spectra of CI Aql and a comparison star, taken with the $4.2 \mathrm{~m}$ William Herschel Telescope on La Palma, on 10th-12th June 2003. A full orbit was observed, including an eclipse. Spectra of a range of spectral type template stars were also taken. The double-armed spectrograph ISIS was used with a $1^{\prime \prime}$ slit width. This gives two wavelength ranges, the red $\lambda \lambda 6120-6750 \AA$ and blue $\lambda \lambda 4570-5370 \AA$ with resolutions of $42.8 \mathrm{~km} \mathrm{~s}^{-1}$ and $55.4 \mathrm{~km} \mathrm{~s}^{-1}$ respectively. The exposure times were $600 \mathrm{~s}$. 
Table 1. System parameters for CI Aql

\begin{tabular}{|c|c|}
\hline Parameter & Determined Value \\
\hline$K_{R}$ & $104 \pm 5 \mathrm{~km} \mathrm{~s}^{-1}$ \\
vsini & $159 \pm 5 \mathrm{~km} \mathrm{~s}^{-1}$ \\
$\Delta \phi_{1 / 2}$ & $0.09 \pm 0.005$ \\
Period & $0.61836348 \pm$ \\
& 0.00000003 days \\
Inclination $i$ & $68^{\circ} \pm 1^{\circ}$ \\
$K_{w}$ & $246 \pm 10 \mathrm{~km} \mathrm{~s}^{-1}$ \\
$M_{W D}$ & $1.02 \pm 0.08 \mathrm{M}_{\odot}$ \\
$M_{2}$ & $2.41 \pm 0.2 \mathrm{M}_{\odot}$ \\
Mass ratio, $q$ & $2.37 \pm 0.15$ \\
$R_{2}$ & $2.10 \pm 0.07 \mathrm{R}_{\odot}$ \\
Separation, $a$ & $4.61 \pm 0.13 \mathrm{R}_{\odot}$ \\
\hline
\end{tabular}

\section{Results and Mass Determination}

In order to determine the system parameters, a number of other measurements are required. The eclipse full-width at half depth, $\Delta \phi_{1 / 2}=0.09 \pm 0.005$, was derived from the red and blue light curves. The radial velocity semi-amplitude, $K_{R}$, and the rotational velocity $v \sin i$, can be determined by an iteration involving cross-correlation (Tonry \& Davis 1979) and optimal subtraction (Smith et al. 1993). The best fitting templates are G4IV in red and F8IV in blue. Using the procedures outlined above gives best fit values of $K_{R}=104 \pm 5 \mathrm{~km} \mathrm{~s}^{-1}$ and $v \sin i=159 \pm 5 \mathrm{~km} \mathrm{~s}^{-1}$. The masses of the two components can now be determined using our results, by adopting the Monte Carlo approach, as set out by Horne et al. (1993). The full results are tabulated in Table 1.

\section{Discussion}

It is possible that CI Aql is evolving into a supersoft X-ray source, with the mass transfer driven by the evolution from the main sequence. The low mass transfer rate at this stage would explain the novae events, but it is expected that the mass transfer rate would increase, leading to steady hydrogen burning on the surface of the WD.

The numerical models of Ivanova \& Taam (2004) show the possible evolutionary fates of systems of main sequence stars and WDs. In Figure 8 from their paper, we have entered the WD and secondary masses for CI Aql and it lies in the region with an ultimate fate of an SN Ia. We therefore suggest that CI Aql will ultimately explode as an SN Ia.

\section{References}

Hachisu, I. \& Kato, M., 2001, ApJ 558:323-350

Horne K., Welsh W. F., \& Wade R. A. 1993, ApJ 410:357-364

Ivanova N. \& Taam R. E. 2004, ApJ 601:1058-1066

Schaefer B. 2001, IAU Circ., 7750, 2

Smith D. A., Dhillon V. S., \& Marsh T. R. 1998, MNRAS, 296, 465-482

Thoroughgood T. D., Dhillon V. S., Littlefair S. P., Marsh T. R., \& Smith D. A. 2001, MNRAS, $327,1323-1333$

Tonry J. \& Davis M. 1979 ApJ 84:1511-1525 